\title{
The neuroprotection effects of exosome in central nervous system injuries: A new target for therapeutic intervention
}

\section{Li Zhang}

Nanjing Jinling Hospital: East Region Military Command General Hospital

Lei Mao

Nanjing Jinling Hospital: East Region Military Command General Hospital

Handong Wang ( $\sim$ njhdwang@hotmail.com)

Nanjing Jinling Hospital: East Region Military Command General Hospital https://orcid.org/00000002-9116-913X

\section{Research Article}

Keywords: Central nervous system injuries, exosomes, downstream molecules

Posted Date: September 20th, 2021

DOI: https://doi.org/10.21203/rs.3.rs-913936/v1

License: (c) (i) This work is licensed under a Creative Commons Attribution 4.0 International License. Read Full License 


\section{Abstract}

Central nervous system (CNS) injuries, including traumatic brain injury (TBI), spinal cord injury (SCl) and subarachnoid hemorrhage (SAH), are the most common cause of death and disability around the world. As a key subset of extracellular vesicles (EVs), exosomes have recently attracted great attentions due to their functions in remodeling extracellular matrix, and transmitting signals and molecules. A large number of studies have suggested that exosomes played an important role in brain development and involved in many neurological disorders, particularly in CNS injuries. It has been proposed that exosomes could improve cognition function, inhibit apoptosis, suppress inflammation, regulate autophagy and protect blood brain barrier (BBB) in CNS injuries via different molecules and pathways including microRNA (miRNA), nuclear factor kappa-light-chain-enhancer of activated B cells (NF-kB), ph1osphatidylinositol4,5-bisphosphate 3-kinase/protein kinase B (PI3K/AKT), Notch1 and extracellular regulated protein kinases (ERK). Therefore, exosomes showed great promise as potential targets in CNS injuries. In this article, we present a review highlighting the applications of exosomes in CNS injuries. Hence, on the basis of these properties and effects, exosomes may be developed as therapeutic agents for CNS injury patients.

\section{Introduction}

Central nervous system (CNS) injuries and their potential long-term consequences are of major concern for public health. High rates of morbidity and mortality making them a global health challenge [1]. CNS is highly sensitive to external mechanical damage, such as traumatic brain injury (TBI), spinal cord injury $(\mathrm{SCl})$, subarachnoid hemorrhage $(\mathrm{SAH})$ and stroke, presenting a limited capacity for regeneration due to its inability to restore either damaged neurons or synaptic network [2]. Although some of the pathological processes of CNS injuries such as blood brain barrier (BBB) disruption, inflammation and oxidative stress have been elucidated, the detailed mechanisms driving these processes are poorly understood [3]. Despite the progress has been made in the prevention and treatment of CNS injuries in the past, patients suffering from CNS injuries usually end up with poor prognosis [4]. Therefore, it is urgently needed to find optimal therapies and improve patients' long-term neurological functioning after CNS injuries.

Extracellular vesicles (EVs) are lipid-bound vesicles that play a significant role in intracellular communication. EVs are classified into three main subtypes including microvesicles (MVs), apoptotic bodies and exosomes. Among EVs, exosomes are most broadly investigated. Exosomes are 30 to $120 \mathrm{~nm}$ endogenous nanovesicles containing proteins, lipids and nucleic acids [5]. The formation of exosomes includes the exocytosis of endosomes and they are secreted out of the cell following the fusion of multivesicular bodies with the plasma membrane (Fig. 1) [6]. Exosomes can cross the BBB and have the potential to specifically deliver molecules to CNS [7]. The initial function of exosomes is thought to be the elimination of non-functional proteins in cells, but the current view is that exosomes are vesicles that are cell-specifically secreted and are involved in intercellular communication [8]. Via cargo proteins, mRNAs, DNAs and miRNAs, exosomes can work locally or be stably transferred to recipient cells and act as key players in triggering, transferring and regulating immune responses to neighbouring cells [9]. Furthermore, 
most cells in CNS have been reported to secrete exosomes into the extracellular environment [10,11]. It has been shown that exosomes were involved in the brain development, functional diversification and contributed to diverse neurological disorders, such as CNS injuries $[12,13]$. In this regard, exosomes could be a promising alternative to cell-based therapies, highlighting the potentially roles of exosomes in CNS injuries are important.

In the present study, we provide an overview of exosomes functions in CNS injuries and the associated molecular mechanisms. This review describes (1) the source of exosomes in CNS injuries; (2) the role of exosomes in CNS injuries; and (3) the downstream targets of exosomes.

\section{The Source Of Exosomes In Cns Injuries}

In 1983, Pan et al. isolated a small vesicle from the supernatant of sheep erythrocytes by ultracentrifugation, the vesicle was later named exosome [14]. This observation led to investigations into the potential role of exosomes in multiple models [15]. Recently, the effects of exosomes in CNS injuries were elucidated. Specifically, exosomes derived from astrocyte, microglia, mesenchymal stem cell (MSC), neuron cells and brain endothelial cells (BECs) were found to influence brain damage in CNS injury models (Table 1).

\subsection{Astrocyte and microglia}

Astrocytes and microglia are able to release cytokines, chemokines and growth factors in respond to brain damage [16]. These factors can affect the homeostatic balance of CNS and determine the degree of injury [17]. Recently, the astrocytes or microglia-derived exosomes have been demonstrated to regulate secondary brain injury after CNS injuries.

Astrocytes are the most abundant glial cells in the CNS. Normally, astrocytes play crucial roles in promoting the formation of BBB, maintaining the function of neural circuit, modulating synaptic circuits and neurotransmitter recycling as well as repairing and scarring process of the brain [18]. In addition to upholding normal brain activities, astrocytes can function as reactive astrogliosis following CNS injuries by regulation of gene expression, morphology and proliferative capacity [19]. Reactive astrogliosis are capable of secreting soluble factors such as transforming growth factor- $\beta$ (TGF- $\beta$ ), vascular endothelial growth factor (VEGF), glial-derived neurotrophic factor (GDNF) and basic fibroblast growth factor (bFGF), which further activated inflammatory response after CNS injuries [20]. The diversity functions of astrocytes make them predominant among other cells in CNS [21,22].

Microglia are brain-resident myeloid cells that regulate immune reaction and inflammatory response [23]. Microglial have been considered as the "gate-keepers" of CNS microenvironment with a large number of functions in development and remodeling of the nervous system [24]. Moreover, microglia modulate cell survival and neurological recovery in response to brain damage by release of trophic factors [25].Upon brain damage, microglia are activated and move toward the lesioned zone, secreting growth factors such as insulin-like growth factor I (IGF-I) and proinflammatory cytokines such as interleukin (IL)-1 $\beta$, IL-6 and 
tumor necrosis factor-a (TNF-a) [26]. Deficiency of microglial has been reported to participate in CNS injuries such as stroke, TBI and hypoxia-ischemia injury $[27,28]$.

\section{$2.2 \mathrm{MSC}$}

MSCs are stromal cells that have the ability to self-renew and also exhibit multilineage differentiation [29]. MSCs were first derived from bone marrow, subsequently they have been isolated from almost all tissues. MSCs can be isolated from adipose tissue, umbilical cord, menses blood and so on [30]. Recently, MSCs have been found in new sources, such as menstrual blood and endometrium [31]. Depending on different parameters such as tissue source, isolation method and medium composition, the role of MSCs are different [32]. MSCs have been an attractive choice for experimental and clinical application because of their relatively simple procedure for cell isolation, self-renewal and capable of differentiating into diverse cell lineages under specific culture conditions [33,34]. Moreover, MSCs can secrete high levels of proteins, cytokines and immune-receptors that functions in immunoregulation, revascularization, cutaneous wound healing, angiogenesis and tissue regeneration [35]. In addition to secreted proteins, MSCs also release exosomes, which could be a promising therapeutic target in diseases [36].

\subsection{Neuron cell}

Neurons are specialized cells with a high level of polarization. The basic function of neurons is responsible for rapid communication of information by receiving, integrating, transmitting and outputting [37]. Neurons consist of three main structural and functional domains: the dendrites, a cell body or soma and an axon. These domains contain smaller compartments, such as the dendritic postsynaptic spines and the axonal presynaptic boutons, for neuronal functions [38,39]. Neurons receive electrochemical signals from other neurons through dendrites, which then communicate information to target cells through the soma and along the axon [40]. Furthermore, neurons are also "excitable" cells, which produce different oscillatory activities that help to understand the transmitting and processing of signals in neural system [41]. The neuronal surface membrane contains ion channels that allow small charged atoms to pass. For example, the activation of potassium or sodium ion channel in neurons can determine firing activities. Therefore, recurrent firing activities as spike trains, sequence of regular or chaotic bursting and mixed-mode type of oscillations may emerge in a single neuron [42].

\subsection{BECs}

BECs are mesodermal derived modified simple squamous epithelial cells that form the walls of blood vessels [43]. A great number of studies have demonstrated that BECs play an important role in brain development, remodeling and repair [44]. Acute injuries, including trauma, cerebral hemorrhage and hypoxia-ischemia, can lead to BEC death, which further causes BBB disruption, inflammation and oxidative stress [45]. Thus, the homeostatic balance of BEC death and survival is vital to brain development and maturation. BECs have unique properties, they lack fenestrations, undergo low rates of transcytosis and are held together by tight junctions (TJs) [46]. These characteristics allow them to limit the vesicle-mediated transcellular movement of solutes and regulate the movement of cells, molecules 
and ions between brain and blood [47]. Moreover, BECs prevent peripheral immune cells to CNS due to their low expression of adhesion molecules [48].

\subsection{Other sources of exosomes}

To date, the four sources of exosome have been well-studied in CNS injuries. However, there were also some other sources of exosome that have been explored such as human urine stem cell (USC) [49], circulating endothelial progenitor cell (cEPC) [50], neural stem cell (NSC) [51], macrophages [52] and dental pulp stem cell (DPSC) [53]. All these sources of exosome may provide neuroprotection in CNS injuries.

\section{The Function Of Exosomes In Cns Injuries}

Exosomes were firstly reported to exhibit neuroprotection on CNS injuries in 2012 [54]. Subsequently, many studies have demonstrated that exosomes could provide neuroprotective effects in CNS injuries. The neuroprotection of exosomes was reportedly attributed to their effects on improvement of cognitive function, inhibition of inflammation, suppression of apoptosis, regulation of autophagy, promotion of angiogenesis and protection of BBB (Table 2).

\subsection{Cognitive function}

In animals, cognitive function is considered to be the ability to learn, retain and recall information. However, in humans, it also represents a complex, multidimensional set of intellectual functions like judgment and evaluation [55]. Thus, in a broader context, cognitive function includes all mental abilities and processes related to knowledge including memory, reasoning, attention, comprehension and language production [56]. Cognitive function was originally thought to be regulated by CNS, but now other systems, for example, the immune system and the intestinal microbiome may also be involved [57]. Cognitive function impairment may occur in CNS injuries and neurodegenerative disease, which is characterized by problems in attention, thinking, memory, language and social communication. Peoples who suffer from cognitive decline experience poor quality of life and demand continuous care from their families and society, thus increasing the burden of family members and social insurance funds [58].

The effects of exosomes on cognitive function after CNS injuries have been explored. In a rat TBI model, MSCs-derived exosomes significant improved spatial learning as measured by the modified Morris water maze test and recovered sensorimotor function as evidenced by reduced neurological deficits and footfault frequency [59]. Furthermore, treatment with exosomes derived from mouse BECs significantly improved neurological and cognitive functional outcome as evaluated by adhesive removal test and odor test in a mouse stroke model [60]. In addition, it has been shown that exosomes from human umbilical cord MSCs attenuated stress-induced hippocampal dysfunctions and improved motor recovery in an acute brain disorder model [61]. 
The precise mechanisms underlying how exosomes regulated cognitive function were unclear. It has been revealed that cognitive function impairment involved selective neuronal loss in the hippocampus and cortex [62]. Therefore, exosomes may also improve cognitive function by intervene with these pathological processes.

\subsection{Inflammation}

Inflammation is one of the major determinants of secondary brain damage after CNS injuries [63]. In normal conditions, inflammation is a vital physiological immune response against noxious stimuli (such as injury or infection) and defends the host against pathogenic threats [64]. However, in respond to CNS injuries, excessive inflammation may provoke substantial detrimental effects. This process involves initiating microglia activation and sustaining astrocytic activation. Once activated, these cells can induce a series of events including activation of glial, recruitment of leukocyte, and release of pro-inflammatory cytokines (e.g., IL-1 $\beta$, IL-2, IL-6, TNF-a, interferon Y (IFN- $\gamma$ )) and chemokines (e.g., C-C motif chemokine ligand 2 (CCL2), C-X-C motif chemokine ligand 8 (CXCL8)) $[65,66]$. These cytokines and chemokines recruit more inflammatory cells to amplify the inflammatory response, leading to BBB breakdown, cerebral edema and cell death [67].

Numerous studies have proposed that exosomes exerted a central effect in CNS injuries-induced inflammation. The effect of exosomes in CNS injuries-induced inflammation was firstly described by Zhang et al. in 2015 [59]. They found that the density of CD68 + and GFAP + cells was significantly increased in the lesion boundary zone after TBI. MSCs-derived exosomes treatment significantly reduced the CD68 + and GFAP + cells density in the injured cortex compared to the phosphate buffered saline (PBS) treatment, suggesting that MSCs-derived exosomes had anti-inflammatory effects in TBI [59]. Moreover, in a mouse model of SAH, bone marrow MSCs-derived exosomes suppressed the expression and activity of histone deacetylase 3 (HDAC3) and up-regulated the acetylation of nuclear factor kappalight-chain-enhancer of activated B cells (NF-KB) p65, thus attenuating neuroinflammation in early brain injury [68]. Furthermore, in ischemic stroke (IS) models, exosomes secreted from the lipopolysaccharide (LPS)-stimulated macrophage promoted microglial polarization from the M1 phenotype to the M2 phenotype and reduced the production of IL-1 $\beta$, TNF-a in vitro, indicating the anti-inflammatory effect of exosomes [52]. In addition, in has been shown that plasma exosomes could enhance melatonin therapeutic effects against ischemia-induced inflammatory responses and inflammasome-mediated pyroptosis in ischemic stroke [69].

The underlying mechanisms of exosomes-mediated inflammation are immensely complicated. Studies have indicated that the NF-KB signaling pathway might be the key target and we will discuss the detailed mechanisms in our following sections.

\subsection{Angiogenesis}

Under physiological conditions, the brain vascular system is stable and contributes to the maintenance and growth of the tissue [70]. When brain vasculature is damage under pathological conditions including injuries, angiogenesis is activated. Angiogenesis is a tightly regulated process through which new blood 
vessels are formed, it involves the participation of endothelial cells, extracellular matrix and vascular cells to form capillaries. This process requires an orchestrated interplay of many stimulators, inhibitors and matrix components [71, 72]. Angiogenesis facilitates the generation of new vasculature, which further accelerates highly coupled neurorestorative process and promotes tissue perfusion. Angiogenesis is controlled by vascular growth factors such as vascular endothelial growth factor (VEGF) [73]. VEGF owns a mitogenic effect on endothelial cells, thus increasing the vascular permeability and promoting cell migration. Besides its role in angiogenesis, VEGF also shows important effects in the neuronal development and physiological function [74]. VEGF can regulate the ion channels of the neuron membrane and accelerate the development of neurons and neural dendritic [75].

Since angiogenesis is benefit for CNS injuries-caused secondary injury, exosomes may attenuate brain damage by promoting angiogenesis. Consistent with this hypothesis, Zhang et al. proposed that MSCsderived exosomes significantly increased the vascular density and angiogenesis as identified by $\mathrm{EBA} / \mathrm{BrdU}+$ double labeling for newborn endothelial cells in the injured cortex [59]. Furthermore, bone marrow MSCs-derived exosomes increased the number of branch points as proven by tube formation assay in a rat hypoxic-ischemic injury model [76]. In another study, it was shown that miRNA-17-92 cluster-enriched exosomes derived from human bone marrow MSCs increased the formation of blood vessels after $\mathrm{TBI}$, indicating that exosomes could promote angiogenesis[77]. Because angiogenesis is emerging as therapeutic target for CNS injuries, therefore, exosomes-based therapies by targeting angiogenesis might provide opportunities for the development of novel therapeutic strategies for CNS injuries.

\subsection{Apoptosis}

Apoptosis is a very tightly programmed cell death (PCD) occurring regularly to eliminate unnecessary and unwanted cells as well as to maintain a homeostatic balance between cell survival and cell death [78]. Apoptosis is critical to animals especially long-lived mammals that must integrate multiple physiological and pathological death signals. Apoptosis therefore represents a physiological and protective response to damage [79]. It has been shown that insufficient apoptosis can trigger cancer or autoimmunity, while excessive activation of apoptosis could be harmful and contribute to abnormal cell death, particularly in pathological conditions such as acute and chronic degenerative diseases, immunodeficiency and trauma [80]. Morphologically, apoptosis renders the cell with shrinkage, which is characterized by DNA fragmentation, chromatin condensation, cytoplasm compacting and plasma membrane blebbing. This is followed by nuclear fragmentation and formation of apoptotic bodies [81]. If apoptosis occurs in CNS injuries, it can cause secondary brain injury, aggravating the damage of brain [82].

The functions of exosomes in apoptosis have been studied. The results obtained by Song et al. demonstrated that microglia-derived exosomes significantly increased cell survival and decreased neuronal apoptosis in ischemia-reperfusion injury, as demonstrated by neuronal survival, TdT-mediated dUTP Nick-End labeling (TUNEL) staining and the lactate dehydrogenase (LDH) assay [83]. In addition, Ni et al. showed that in a mouse TBI model, bone marrow MSCs-derived exosomes up-regulated the expression of B-cell lymphoma-2 (Bcl-2) while down-regulated the expressions of $\mathrm{Bcl}$-2-associated $\mathrm{X}$ 
protein (Bax), suggesting that bone marrow MSCs-exosomes attenuated cell apoptosis [84]. In another study conducted by Lai et al., they found that MSCs-derived exosomes decreased apoptosis in brain following SAH as shown by increased expression of $\mathrm{Bcl}-2$ and decreased expression of caspase-3 [68]. In conclusion, these data suggested that exosomes could reduce cell apoptosis in models of CNS injury.

Researches so far have only studied the role of exosomes on apoptosis in general. However, apoptosis can be divided into two pathways: the mitochondria-dependent pathway (the intrinsic pathway) and the death receptor-dependent pathway (the extrinsic pathway). The intrinsic pathway involves a chain of intracellular events occurring in the mitochondrion including the release of cytochrome $c$, formation of the apoptosome with apoptotic protease-activating factor 1 (APAF1), activation of caspase- 9 and subsequent caspase-3. The release of cytochrome $\mathrm{c}$ is positively regulated by the pro-apoptotic $\mathrm{Bcl}-2$ family members such as Bax, Bcl-2 antagonist killer 1 (Bak), Bid and negatively regulated by the antiapoptotic Bcl-2 family members such as Bcl-2, B-cell lymphoma-extra large (Bcl-xL). In contrast, the extrinsic pathway is initiated by the binding of TNF ligand to TNF receptor and the binding of Fas ligand to Fas receptor. Upon ligand binding, the death receptors allow the binding of an initiator caspase-8 or -10 to form death inducing signaling complex (DISC) through its death effector domain (DED). The activation of caspase-8 relays the death signal to an execution caspase to bring about apoptosis $[85,86]$. Thus, which apoptotic pathway is associated with the effects of exosomes in CNS injuries-induced apoptosis remains unclear and further studies are needed to clarify it.

\subsection{Autophagy}

Autophagy is an evolutionarily conserved lysosomal pathway for the degradation of cytoplasmic components [87]. In conditions of starvation response, cell differentiation and quality control, autophagy is activated and plays an important role in maintaining and regulating cell homeostasis by degrading intracellular components and providing degradation products to cells [88]. Autophagy begins with the formation of a membrane vesicle called the phagophore, which matures into a spherical lipid bilayer vesicle named the autophagosome. The autophagosome then fuses with a lysosome and degrades the contents in autolysosome [89]. Recent studies have revealed that the dysfunction of autophagy was implicated in CNS injuries and extensive activation of autophagy can lead to type II PCD [90]. Up to now, the dual role of autophagy in protective or destructive of CNS injuries remains controversial. Shi et al. found that in cerebral ischemia-reperfusion rats, inhibiting autophagy by sevoflurane attenuated brain damage, demonstrating a detrimental role of autophagy [91]. Conversely, Ahsan et al. reported that Urolithin A-activated autophagy protected against ischemic neuronal injury by inhibiting endoplasmic reticulum (ER) stress both in vitro and in vivo, suggesting that autophagy played a beneficial role in stroke [92].

There were also studies showing that exosomes could affect autophagy in CNS injuries. However, the roles of exosomes-regulated autophagy in CNS injuries were also controversial. Li et al. have shown that exosomes from neurons inhibited cell apoptosis and death in TBI by suppression of Rab11a-mediated autophagy, suggesting a detrimental role of autophagy in TBI [93]. Interestingly, in another study conducted by Yuan et al., they found that bone marrow MSCs-derived exosomes decreased ER stress in 
BV2 cells by induction of disabled homolog 2-interacting protein (DAB2IP)-mediated microglial cell autophagy, suggesting a protective role of exosomes and autophagy in brain injury [94]. The discrepancies may be due to the different source of exosomes and cell types used in these two studies. Taken together, by combination with the previous studies, we though that depending on different CNS injury models, sources of exosomes and cell types, autophagy and cell death may have inhibitory, additive or even synergistic effects.

\subsection{BBB function}

BBB is a highly specialized, semi-permeable physical barrier that locates at the interface between the CNS and the surrounding environment. It is instrumental in regulating the metabolism of brain, maintaining the microenvironmental homeostasis of CNS and coordinating the functions of peripheral organs [95]. In addition, BBB is a dynamic metabolic interface that can bi-directionally regulate the transport of fluids, solutes and cells [96]. Structurally, BBB is formed by BECs with TJ. Dysfunction of BBB is a common pathological feature in CNS injuries. Several underlying events are involved in BBB destruction, such as disruption of the TJ, breakdown of the BECs and degradation of the extracellular matrix [97]. In an in vitro model of IS, Pan et al. found that MSCs-derived exosomes alleviated BBB disruption in hypoxia/reoxygenation (H/R)-injured endothelial cells by analyzing the Evans blue dye extravasation and brain water content [98]. Moreover, Lai et al. suggested that bone marrow MSCs-derived exosomes attenuated BBB permeability in early brain injury after SAH [68]. Furthermore, another in vivo study confirmed the protective effects of exosomes on BBB in ischemia-reperfusion injury [99].

\section{Downstream Molecules Of Exosomes In Cns Injuries}

The specific mechanisms mediating the functions of exosomes in CNS injuries have yet to be fully explained, a number of downstream molecules of exosomes have been suggested which may explain their biological effects (Fig. 2).

\subsection{MicroRNA (miRNA)}

MiRNAs, a subset of non-coding RNAs, are 19 to 25 nucleotide long endogenously-initiated short RNA molecules [100]. MiRNAs modulate gene expression at the post-transcriptional level via translational inhibition or messenger RNA (mRNA) degradation and control a range of biological functions, including developmental timing and host-pathogen interactions as well as cell proliferation, apoptosis and tumorigenesis [101]. As a regulatory element, miRNA itself is also regulated by multiple effectors such as miRNA editing, circadian clock and methylation. However, illustrating the exact roles of miRNAs in these pathophysiological processes are difficult because of their complexity of actions [102]. Formation of a miRNA consists of two steps, from primary (pri) miRNA to precursor (pre) miRNAs mediated by Drosha in the nucleus and from pre-miRNAs to mature miRNAs mediated by Dicer in the cytoplasm [103].

MiRNAs contribute to nearly $1 \%$ of all predicted genes in nematodes, flies and mammals. Because of their simple structure and ability to modulate cellular functions, an expanding body of evidences have 
shown that miRNA-based therapies, either activating or inhibiting, hold great promise [104]. Recently, miRNAs have been identified in exosomes, which can be taken up by neighboring or distant cells and subsequently modulate recipient cells. Exosomal miRNAs play an important role in disease progression and provide neuroprotection in CNS injuries by stimulating angiogenesis, suppressing apoptosis and reduced inflammation. It has been shown that microglia-derived exosomes protected the mouse brain from ischemia-reperfusion injury via exosomal miRNA-124 [83]. Moreover, in a rat hypoxic-ischemic injury model, bone marrow MSCs-derived exosomal miRNA-29b-3p promoted angiogenesis and suppressed apoptosis in the brain [76]. Furthermore, MSCs-derived exosomal miRNA-193b-3p attenuated neuroinflammation in early brain injury after SAH [68].

Among exosomal cargo biomolecules, miRNAs obtain the most attention due to their regulative effects in gene expression. Studies have shown that miRNAs are not randomly incorporated into exosomes, a subset of miRNAs may preferentially enter exosomes [105]. Although the precise mechanisms of sorting miRNAs into exosomes remain unclear so far, four potential modes have been suggested. These include: (1) The neural sphingomyelinase 2 (nSMase2)-dependent pathway. (2) The miRNA motif and sumoylated heterogeneous nuclear ribonucleoproteins (hnRNPs)-dependent pathway. (3) The 3'-end of the miRNA sequence-dependent pathway. (4) The miRNA induced silencing complex (miRISC)-related pathway [106]. However, further studies are needed to prove which mode is associated with the incorporation of miRNAs into exosomes in CNS injuries.

\subsection{Nuclear factor kappa-light-chain-enhancer of activated B cells (NF-KB)}

NF-KB is a family of dimeric transcription factors that involved in inflammatory responses, innate and adaptive immunity as well as cell proliferation and differentiation [107]. NF-KB can protect cells against inflammation and cell death by regulating the transcription of genes including cytokines, chemokines and adhesion molecules [108]. There are mainly two pathways for NF-KB activation, the "canonical" pathway and the "alternative" pathway. The "canonical" pathway is triggered by microbial products and proinflammatory cytokines such as IL-1 and TNF-a, leading to the activation of RelA- or cRel- containing complexes. The "alternative" pathway is initiated by TNF-family cytokines, B cell activating factor and receptor activator of NF-KB ligand, resulting in activation of RelB/p52 complexes $[109,110]$.

$\mathrm{NF}-\mathrm{kB}$ is tightly regulated, and activation of NF-KB has been implicated in CNS injuries. Exosomes were also shown to modulate inflammatory response in CNS injuries by activation of NF-KB. In ischemiareperfusion injury models, the inflammatory response reflexed by the levels of TNF-a, IL-1 $\beta$, IL- 6 and their mediator, NF-KB were suppressed by DPSCs-derived exosomes [53]. Furthermore, in in vivo and in vitro SCl models, NSCs-derived exosomes downregulated the NF-KB-p65 axis in rats and PC12 cells respectively, thus inhibiting the inflammatory response [51]. Similar results were found in SCI models using bone marrow MSCs-derived exosomes both in vivo and in vitro [111]. Therefore, functional exosomes are important to regulate inflammation via regulation of NF-KB in CNS injuries. 
How exosomes regulated NF-KB in CNS injuries have not been well characterized. There were reports showing that long noncoding RNAs (IncRNAs) and miRNAs may be involved. For example, stem cellderived exosomes could prevent aging-induced cardiac dysfunction through the IncRNA MALAT1/NF-KB signaling pathway [112]. The regulation of NF-KB by IncRNAs can be mediated by the interaction between IncRNAs and the p65 subunit of NF-KB. LncRNAs bind with the p65 subunit of NF-KB and IkB to form a stable IncRNAs/NF-kB/lkB complex. Then, the phosphorylation sited of $\mathrm{lkB}$ is masked, thus inhibiting $\mathrm{lkB}$ kinases (IKK)-induced IkB phosphorylation and NF-KB activation [113]. In addition, it has been shown that miRNAs could regulate NF-KB by interfere with the signaling components upstream of NF-KB such as affecting the phosphorylation of IKK and IKB. For example, activation of miR-214-3p decreased cell apoptosis and inflammation in osteoarthritis (OA) by downregulated the IKK- $\beta$ expression and led to the dysfunction of NF-KB signaling pathway [114]. Therefore, we speculated that exosomes might also regulate NF-kB via IncRNAs and miRNAs in CNS injuries. However, further studies were needed to confirm our hypothesis.

\subsection{Phosphatidylinositol-4,5-bisphosphate 3-kinase/protein kinase B (PI3K/AKT) pathway}

The PI3K/AKT is an intracellular signaling pathway that participates in a broad range of cellular processes including cell proliferation, differentiation, metabolism and quiescence [115]. The PI3K/AKT pathway can be activated by ligands, including cytokines, hormones and growth factors. These ligands further bind to receptors, such as epidermal growth factor receptor (EGFR), receptor tyrosine kinase (RTK) and G-protein-coupled receptor (GPCR). Activation of these receptors firstly activates class I PI3K, then phosphorylates phosphatidylinositol 4,5-biphosphate (PIP2) to phosphatidylinositol 3,4,5-triphosphate (PIP3) and recruits signaling proteins, including AKT [116].The PI3K/AKT pathway can also be activated by loss of phosphatase and tensin homolog (PTEN). PTEN is a main negative regulator of the PI3K that dephosphorylates PIP3 to PIP2 [117].

Exosomes have been found to exhibit protective effects in CNS injuries by activation of the PI3K/AKT pathway. It has been identified that MSCs-derived exosomes decreased ROS production, apoptosis and TJ disruption in H/R-injured endothelial cells. Moreover, MSCs-derived exosomes activated PI3K/AKT pathway and inhibition of PI3K by its inhibitor LY294002 ameliorated the protective effects of exosomes [98]. Moreover, Cao et al. revealed that USC-derived exosomes harboring ANGPTL3 enhanced spinal cord functional recovery after SCl by activation of the PI3K/AKT pathway [49]. Furthermore, Wang et al. indicated that cEPCs-derived exosomes had beneficial effects on mouse IS by attenuating infarct volume and cell apoptosis, increasing the microvessel density and promoting axon growth ability via activating the PI3K/AKT pathway [50].

But how exosomes regulated the PI3K/AKT pathway in CNS injuries was uncertain. Recently, in many cancer models, it has been proposed that the regulation of the PI3K/AKT pathway by exosomes might be associated with the miRNA/PTEN pathway [118-120]. That means, exosomes firstly controlled miRNAs, which further regulated PTEN and the downstream PI3K/AKT pathway. For example, colorectal cancer 
(CRC) cell-derived exosomal miRNA-934 induced M2 macrophage polarization by downregulating PTEN expression and activating the PI3K/AKT signaling pathway [121]. In another case, exosomal miRNA-223 derived from macrophages promoted the drug resistance of epithelial ovarian cancer (EOC) cells via the PTEN-PI3K/AKT pathway. In addition, exosomal miRNA-32-5p induced multidrug resistance in hepatocellular carcinoma by down-regulation of PTEN to activate the PI3K/AKT signaling pathway [122]. Therefore, combined with these literatures, we speculated that exosomes may also regulate the PI3K/AKT pathway via miRNAs-PTEN axis in CNS injuries. Further studies are needed to explore it.

\subsection{Notch1}

Notch1 is a class I transmembrane protein that directly transduces extracellular signals into cells. Notch1 modulates interactions between physically adjacent cells and plays an essential role in cell fate decisions and tissue homeostasis by binding to its ligands [123]. Notch1 signaling is initiated by interaction of the receptor with its ligands Delta-1 or Jagged-1. This ligand-receptor interaction triggers Notch1 intracellular cytoplasmic domain (NICD) which translocates into the nucleus. There, nuclear Notch1 associates with the transcription factor recombining binding protein suppressor of hairless (RBP-JK), activates the expression of target genes and regulates a variety of cellular metabolisms [124]. Recent studies have demonstrated that the Notch1 signaling pathway was involved in CNS injuries such as cerebral ischemic injury. Blockage of the Notch1 signaling pathway could reduce neuronal cell apoptosis, suppress inflammatory response, promote angiogenesis and improve prognosis in CNS injuries $[125,126]$. Exosomes also facilitated Notch1 to provide neuroprotection in ischemic brain injury. It has been suggested that down-regulation of Notch 1 induced by microglia-derived exosomes was associated with decreased neurobehavioral deficits, fewer infarct areas in the brain and less apoptosis in ischemiareperfusion injury. In addition, the Notch1 inhibitor Cren further enhanced the effects [127].

The underlying mechanism of how exosomes regulate Notch1 may involve miRNAs. It has been shown that miRNA-137 could mediate the function of microglia-derived exosomes by binding to the 3' untranslated region (UTR) of Notch1 [127]. In another case, Liu et al. found that in bone marrow MSCs, exosomes secreted by mesenchymal stem cell transplantation (MSCT) reduced intracellular levels of miR-29b, which resulted in recovery of DNA methyltransferase 1 (Dnmt1)-mediated Notch1 promoter hypomethylation and inhibition of Notch1 signaling [128]. These data indicated a critical role of miRNAs between exosomes and Notch1 signaling. However, the clear mechanisms of how exosomes regulate Nothch1 in CNS injuries are unknow, which is an interesting aspect worth exploring.

The Notch family consists of four receptors, classified as Notch1, Notch2, Notch3, and Notch4 receptors. Notch1 and Notch2 are expressed widely in tissues throughout development. Notch3 is expressed mainly in pericytes and vascular smooth muscle, and Notch4 is most abundant in endothelium [129]. The distribution differences make the functions of the Notch receptors variants rather distinct. Notch1 mutation leads to vascular malformations, Notch2 mutation causes vascular and renal defects, Notch3 mutation induces viable and fertile and Notch4 is dispensable for embryonic development [130]. Since Notch 1 can be controlled by exosomes in ischemic brain injury, further studies are needed to clarify whether other Notch receptors such as Notch2 and Notch3 can be regulated by exosomes in CNS injuries. 


\subsection{Extracellular signal-regulated kinase (ERK)}

ERK is a serine/threonine protein kinase that belongs to the mitogen-activated protein kinase (MAPK) family It is widely expressed in eukaryotic cells [131]. Various stimuli, such as trauma, oxidative stress and inflammation, activate tyrosine kinases and transmit the signal to rat sarcoma virus (Ras). Rasguanosine triphosphate (GTP) binds to rapidly accelerated fibrosarcoma (Raf) and induces its translocation to cell membrane from cytoplasm. Activated Raf phosphorylates serine residues of mitogen-activated protein kinase kinase (MEK) in its catalytic region, which further phosphorylates ERK. Phosphorylated ERK moves to the nucleus, activates many transcription factors, and results in regulation of cell proliferation, differentiation, gene expression and various physiological processes [132]. In physiological states, ERK is essential for normal development and functional plasticity of the CNS. However, in pathological states such as cerebral ischemia, brain trauma and ischemia-reperfusion injury, abnormally expression of ERK may play a detrimental role by promoting cell apoptosis and oxidative stress [133]. Exosomes have been shown to regulate ERK by affecting its phosphorylation. Long et al. implied that astrocyte-derived exosomes significantly inhibited lipopolysaccharide (LPS)-induced microglial M1 phenotype transformation and the subsequent inflammation through decreased phosphorylation of ERK [134].

\section{Other Aspects Of Exosome Research In Cns Injuries}

CNS injuries, caused by cerebrovascular pathologies or mechanical contusions, comprise a diverse group of pathological processes, including glutamate excitotoxicity, oxidative stress, apoptosis and autophagy [135]. Although the functions of exosomes on CNS injuries-induced cognitive function, inflammation, angiogenesis, apoptosis, autophagy and BBB disruption have been widely described, its roles in excitotoxicity and oxidative stress have not been fully illustrated.

\subsection{Excitotoxicity}

Excitotoxicity is a phenomenon that describes the damage of cells due to exacerbated exposure to excitatory amino acids [136]. The underlying mechanisms of excitotoxicity includes alterations in glutamate and $\mathrm{Ca}^{2+}$ metabolism, dysfunction of glutamate transporters and malfunction of glutamate receptors [137]. In this process, glutamate is the main factor that induces excitotoxic cell damage. Normally, glutamate plays crucial roles in neuronal growth, axon guidance and synaptic plasticity. However, excessive or prolonged activation of glutamate causes the imbalance of neuronal $\mathrm{Ca}^{2+}$ homeostasis and final excitotoxicity, leading to mitochondrial destruction, neuronal damage and oxidative stress [138].

The functions of exosomes in excitotoxicity have also been well established. It has been shown that longterm secretion of exosomes protected neurons from excitotoxic damage in the model of trophic factors deprivation [139]. Besides, astrocyte-derived exosomes suppressed glutamate-induced hippocampal neuron death in an in vitro glutamate excitotoxicity model [140]. In addition, in Alzheimer's disease (AD) 
models, exosomes isolated from AD patient cerebrospinal fluid (CSF) and plasma, from the plasma of AD mouse models, and from the medium of neural cells expressing familial $A D$ presenilin 1 mutation impaired neuronal $\mathrm{Ca}^{2+}$ handling and mitochondrial function, and render neurons vulnerable to excitotoxicity [141]. Therefore, exosomes may also intervene excitotoxicity in CNS injuries. However, further studies are needed to verify it.

\subsection{Oxidative stress}

Oxidative stress, defined as imbalance between the biological systems leading to the generation of oxidant (free) radicals and the systems responsible for the removal of free radicals, is harmful to cells due to the excessive generation of oxidant compounds such as reactive oxygen species (ROS) and reactive nitrogen species (RNS) [142]. Under physiological conditions, both ROS and RNS are generated at moderate concentrations and act as second messengers to regulate signal transduction pathways. However, the excessive generation of ROS and RNS due to depletion of the antioxidant system or excitotoxicity leads to the oxidation of biological molecules such as lipids, proteins, and DNA, resulting in oxidative damage in cells, tissues and organs [143]. Oxidative stress has been reported in CNS injury models and contributed to the secondary brain damage such as brain edema, BBB damage and apoptosis $[144,145]$.

There were also researches indicating that exosomes could regulate oxidative stress. Wang et al. suggested that MSCs-derived exosomes could protect against oxidative stress-induced skin injury via adaptive regulation of the nuclear factor erythroid 2-related factor 2 (Nrf2) defense system [146]. Moreover, Arslan et al. proposed that MSCs-derived exosomes increased adenosine triphosphate (ATP) levels, decreased oxidative stress, enhanced myocardial viability and prevented adverse remodeling after myocardial ischemia/reperfusion injury by activating the PI3K/AKT pathway [147]. In addition, strawberry-derived exosomes prevented oxidative stress in human mesenchymal stromal cells [148]. Therefore, whether exosomes could regulate oxidative stress in CNS injuries needed to be further studied.

\section{Concluding Remarks}

Exosomes play essential roles in CNS injuries and participate in a number of cellular and molecular processes of CNS injuries. In this review, we summarize the sources of exosomes, the functions of exosomes as well as some downstream moleculars of exosomes in CNS injuries. These observations make exosomes to be attractive therapeutic targets for patients suffering from CNS injuries. Continued discoveries in this field will bring novel insights on exosomes involved in biological functions and disease progression. Ultimately, exosomes may hold promise for clinical challenges.

\section{Declarations}

Funding 
This work was supported by Grants from the construction of key medical disciplines in Jiangsu Province (No. ZDXKB2016023) from Handong Wang.

\section{Conflict of interest statement}

The authors declare no conflict of interest.

\section{Availability of data and material}

All data generated during this review are included in this article.

\section{Code availability}

Not applicable.

\section{Authors' contributions}

Professor Handong Wang conceived the whole work design and played a vital role in paper submission.

Li Zhang finished the original manuscript including figures and tables. Lei Mao revised the manuscript.

Additional declarations for articles in life science journals that report the results of studies involving humans and/or animals

Not applicable.

\section{Ethics approval}

Not applicable.

\section{Consent to participate}

Not applicable.

\section{Consent for publication}

Not applicable.

\section{Acknowledgements}

Not applicable.

\section{References}

1. Hornby TG, Reisman DS, Ward IG, Scheets PL, Miller A, Haddad D, Fox EJ, Fritz NE, Hawkins K, Henderson CE, Hendron KL, Holleran CL, Lynskey JE, Walter A, and the Locomotor CPGAT (2020) Clinical Practice Guideline to Improve Locomotor Function Following Chronic Stroke, Incomplete 
Spinal Cord Injury, and Brain Injury. Journal of neurologic physical therapy: JNPT 44 (1):49-100. doi:10.1097/NPT.0000000000000303

2. Zhang L, Wang H (2019) Long Non-coding RNA in CNS Injuries: A New Target for Therapeutic Intervention. Molecular therapy Nucleic acids 17:754-766. doi:10.1016/j.omtn.2019.07.013

3. Devanney NA, Stewart AN, Gensel JC (2020) Microglia and macrophage metabolism in CNS injury and disease: The role of immunometabolism in neurodegeneration and neurotrauma. Exp Neurol 329:113310. doi:10.1016/j.expneurol.2020.113310

4. Liddelow SA, Barres BA (2017) Reactive Astrocytes: Production, Function, and Therapeutic Potential. Immunity 46(6):957-967. doi:10.1016/j.immuni.2017.06.006

5. Jiang L, Dong H, Cao H, Ji X, Luan S, Liu J (2019) Exosomes in Pathogenesis, Diagnosis, and Treatment of Alzheimer's Disease. Medical science monitor: international medical journal of experimental clinical research 25:3329-3335. doi:10.12659/MSM.914027

6. Gurunathan S, Kang MH, Jeyaraj M, Qasim M, Kim JH (2019) Review of the Isolation, Characterization, Biological Function, and Multifarious Therapeutic Approaches of Exosomes. Cells 8 (4). doi:10.3390/cells8040307

7. Liu J, Jiang F, Jiang Y, Wang Y, Li Z, Shi X, Zhu Y, Wang H, Zhang Z (2020) Roles of Exosomes in Ocular Diseases. Int J Nanomed 15:10519-10538. doi:10.2147/IJN.S277190

8. Li XH, Zhang J, Li DF, Wu W, Xie ZW, Liu Q (2020) Physiological and pathological insights into exosomes in the brain. Zoological research 41(4):365-372. doi:10.24272/j.issn.2095-8137.2020.043

9. Levy E (2017) Exosomes in the Diseased Brain: First Insights from In vivo Studies. Front NeuroSci 11:142. doi:10.3389/fnins.2017.00142

10. Gharbi T, Zhang Z, Yang GY (2020) The Function of Astrocyte Mediated Extracellular Vesicles in Central Nervous System Diseases. Frontiers in cell developmental biology 8:568889. doi:10.3389/fcell.2020.568889

11. Chen J, Chopp M (2018) Exosome Therapy for Stroke. Stroke 49(5):1083-1090. doi:10.1161/STROKEAHA.117.018292

12. Zhang ZG, Buller B, Chopp M (2019) Exosomes - beyond stem cells for restorative therapy in stroke and neurological injury. Nature reviews Neurology 15(4):193-203. doi:10.1038/s41582-018-0126-4

13. Liu W, Bai X, Zhang A, Huang J, Xu S, Zhang J (2019) Role of Exosomes in Central Nervous System Diseases. Front Mol Neurosci 12:240. doi:10.3389/fnmol.2019.00240

14. Minetti G, Achilli C, Perotti C, Ciana A (2018) Continuous Change in Membrane and MembraneSkeleton Organization During Development From Proerythroblast to Senescent Red Blood Cell. Frontiers in physiology 9:286. doi:10.3389/fphys.2018.00286

15. Guedes VA, Devoto C, Leete J, Sass D, Acott JD, Mithani S, Gill JM (2020) Extracellular Vesicle Proteins and MicroRNAs as Biomarkers for Traumatic Brain Injury. Front Neurol 11:663. doi:10.3389/fneur.2020.00663 
16. Sacristan C (2020) Microglia and Astrocyte Crosstalk in Immunity. Trends Immunol 41(9):747-748. doi:10.1016/j.it.2020.07.009

17. Guttenplan KA, Liddelow SA (2019) Astrocytes and microglia: Models and tools. The Journal of experimental medicine 216(1):71-83. doi:10.1084/jem.20180200

18. Sofroniew MV (2020) Astrocyte Reactivity: Subtypes, States, and Functions in CNS Innate Immunity. Trends Immunol 41(9):758-770. doi:10.1016/j.it.2020.07.004

19. Zhou B, Zuo YX, Jiang RT (2019) Astrocyte morphology: Diversity, plasticity, and role in neurological diseases. CNS Neurosci Ther 25(6):665-673. doi:10.1111/cns.13123

20. Spiranec Spes K, Hupp S, Werner F, Koch F, Volker K, Krebes L, Kammerer U, Heinze KG, Braunger BM, Kuhn M (2020) Natriuretic Peptides Attenuate Retinal Pathological Neovascularization Via Cyclic Guanosine Monophosphate Signaling in Pericytes and Astrocytes. Arteriosclerosis, thrombosis, and vascular biology 40 (1):159-174. doi:10.1161/ATVBAHA.119.313400

21. Pekny M, Wilhelmsson U, Tatlisumak T, Pekna M (2019) Astrocyte activation and reactive gliosis-A new target in stroke? Neurosci Lett 689:45-55. doi:10.1016/j.neulet.2018.07.021

22. Michinaga S, Koyama Y (2019) Dual Roles of Astrocyte-Derived Factors in Regulation of Blood-Brain Barrier Function after Brain Damage. Int J Mol Sci 20 (3). doi:10.3390/ijms20030571

23. Li D, Lang W, Zhou C, Wu C, Zhang F, Liu Q, Yang S, Hao J (2018) Upregulation of Microglial ZEB1 Ameliorates Brain Damage after Acute Ischemic Stroke. Cell reports 22(13):3574-3586. doi:10.1016/j.celrep.2018.03.011

24. Masuda T, Amann L, Sankowski R, Staszewski O, Lenz M, Snaidero PDE, Costa Jordao N, Bottcher MJ, Kierdorf C, Jung K, Priller S, Misgeld J, Vlachos T, Meyer-Luehmann A, Knobeloch M, Prinz KP M (2020) Novel Hexb-based tools for studying microglia in the CNS. Nature immunology 21(7):802815. doi:10.1038/s41590-020-0707-4

25. Chen Z, Zhong D, Li G (2019) The role of microglia in viral encephalitis: a review. J Neuroinflamm 16(1):76. doi:10.1186/s12974-019-1443-2

26. Sominsky L, De Luca S, Spencer SJ (2018) Microglia: Key players in neurodevelopment and neuronal plasticity. Int J Biochem Cell Biol 94:56-60. doi:10.1016/j.biocel.2017.11.012

27. Tsuda M (2018) Microglia in the CNS and Neuropathic Pain. Adv Exp Med Biol 1099:77-91. doi:10.1007/978-981-13-1756-9_7

28. Rodriguez-Gomez JA, Kavanagh E, Engskog-Vlachos P, Engskog MKR, Herrera AJ, Espinosa-Oliva AM, Joseph B, Hajji N, Venero JL, Burguillos MA (2020) Microglia: Agents of the CNS Pro-Inflammatory Response. Cells 9 (7). doi:10.3390/cells9071717

29. Mansoor SR, Zabihi E, Ghasemi-Kasman M (2019) The potential use of mesenchymal stem cells for the treatment of multiple sclerosis. Life sciences 235:116830. doi:10.1016/j.Ifs.2019.116830

30. Li Z, Liu F, He X, Yang X, Shan F, Feng J (2019) Exosomes derived from mesenchymal stem cells attenuate inflammation and demyelination of the central nervous system in EAE rats by regulating the polarization of microglia. Int Immunopharmacol 67:268-280. doi:10.1016/j.intimp.2018.12.001 
31. Bonsack B, Corey S, Shear A, Heyck M, Cozene B, Sadanandan N, Zhang H, Gonzales-Portillo B, Sheyner M, Borlongan CV (2020) Mesenchymal stem cell therapy alleviates the neuroinflammation associated with acquired brain injury. CNS Neurosci Ther 26(6):603-615. doi:10.1111/cns.13378

32. Gorabi AM, Kiaie N, Barreto GE, Read MI, Tafti HA, Sahebkar A (2019) The Therapeutic Potential of Mesenchymal Stem Cell-Derived Exosomes in Treatment of Neurodegenerative Diseases. Mol Neurobiol 56(12):8157-8167. doi:10.1007/s12035-019-01663-0

33. Shahror RA, Wu CC, Chiang YH, Chen KY (2020) Genetically Modified Mesenchymal Stem Cells: The Next Generation of Stem Cell-Based Therapy for TBI. Int J Mol Sci 21 (11). doi:10.3390/ijms21114051

34. Branscome H, Paul S, Khatkar P, Kim Y, Barclay RA, Pinto DO, Yin D, Zhou W, Liotta LA, El-Hage N, Kashanchi F (2020) Stem Cell Extracellular Vesicles and their Potential to Contribute to the Repair of Damaged CNS Cells. Journal of neuroimmune pharmacology: the official journal of the Society on Neurolmmune Pharmacology 15(3):520-537. doi:10.1007/s11481-019-09865-y

35. Samper Agrelo I, Schira-Heinen J, Beyer F, Groh J, Butermann C, Estrada V, Poschmann G, Bribian A, Jadasz JJ, Lopez-Mascaraque L, Kremer D, Martini R, Muller HW, Hartung HP, Adjaye J, Stuhler K, Kury P (2020) Secretome Analysis of Mesenchymal Stem Cell Factors Fostering Oligodendroglial Differentiation of Neural Stem Cells In Vivo. Int J Mol Sci 21 (12). doi:10.3390/ijms21124350

36. Branscome H, Paul S, Yin D, El-Hage N, Agbottah ET, Zadeh MA, Liotta LA, Kashanchi F (2020) Use of Stem Cell Extracellular Vesicles as a "Holistic" Approach to CNS Repair. Frontiers in cell developmental biology 8:455. doi:10.3389/fcell.2020.00455

37. Carroll JA, Chesebro B (2019) Neuroinflammation, Microglia, and Cell-Association during Prion Disease. Viruses 11 (1). doi:10.3390/v11010065

38. Miller MW (2019) GABA as a Neurotransmitter in Gastropod Molluscs. Biol Bull 236(2):144-156. doi:10.1086/701377

39. Song I, Dityatev A (2018) Crosstalk between glia, extracellular matrix and neurons. Brain research bulletin 136:101-108. doi:10.1016/j.brainresbull.2017.03.003

40. Habermacher C, Angulo MC, Benamer N (2019) Glutamate versus GABA in neuron-oligodendroglia communication. Glia 67(11):2092-2106. doi:10.1002/glia.23618

41. Rubio C, Taddei E, Acosta J, Custodio V, Paz C (2020) Neuronal Excitability in Epileptogenic Zones Regulated by the Wnt/ Beta-Catenin Pathway. CNS Neurol Disord Drug Target 19(1):2-11. doi:10.2174/1871527319666200120143133

42. Ohura S, Kamiya H (2016) Excitability tuning of axons in the central nervous system. J Physiol Sci 66(3):189-196. doi:10.1007/s12576-015-0415-2

43. Abbott NJ, Patabendige AA, Dolman DE, Yusof SR, Begley DJ (2010) Structure and function of the blood-brain barrier. Neurobiol Dis 37(1):13-25. doi:10.1016/j.nbd.2009.07.030

44. Castro Dias M, Mapunda JA, Vladymyrov M, Engelhardt B (2019) Structure and Junctional Complexes of Endothelial, Epithelial and Glial Brain Barriers. Int J Mol Sci 20 (21). doi:10.3390/ijms20215372 
45. Saint-Pol J, Gosselet F, Duban-Deweer S, Pottiez G, Karamanos Y (2020) Targeting and Crossing the Blood-Brain Barrier with Extracellular Vesicles. Cells 9 (4). doi:10.3390/cells9040851

46. Yazdani S, Jaldin-Fincati JR, Pereira RVS, Klip A (2019) Endothelial cell barriers: Transport of molecules between blood and tissues. Traffic 20(6):390-403. doi:10.1111/tra.12645

47. Minami T, Muramatsu M, Kume T (2019) Organ/Tissue-Specific Vascular Endothelial Cell Heterogeneity in Health and Disease. Biol Pharm Bull 42(10):1609-1619. doi:10.1248/bpb.b1900531

48. Nishihara H, Gastfriend BD, Kasap P, Palecek SP, Shusta EV, Engelhardt B (2021) Differentiation of human pluripotent stem cells to brain microvascular endothelial cell-like cells suitable to study immune cell interactions. STAR protocols 2(2):100563. doi:10.1016/j.xpro.2021.100563

49. Cao Y, Xu Y, Chen C, Xie H, Lu H, Hu J (2021) Local delivery of USC-derived exosomes harboring ANGPTL3 enhances spinal cord functional recovery after injury by promoting angiogenesis. Stem Cell Res Ther 12(1):20. doi:10.1186/s13287-020-02078-8

50. Wang J, Liu H, Chen S, Zhang W, Chen Y, Yang Y (2020) Moderate exercise has beneficial effects on mouse ischemic stroke by enhancing the functions of circulating endothelial progenitor cell-derived exosomes. Exp Neurol 330:113325. doi:10.1016/j.expneurol.2020.113325

51. Ma K, Xu H, Zhang J, Zhao F, Liang H, Sun H, Li P, Zhang S, Wang R, Chen X (2019) Insulin-like growth factor-1 enhances neuroprotective effects of neural stem cell exosomes after spinal cord injury via an miR-219a-2-3p/YY1 mechanism. Aging 11(24):12278-12294.

doi:10.18632/aging.102568

52. Zheng Y, He R, Wang P, Shi Y, Zhao L, Liang J (2019) Exosomes from LPS-stimulated macrophages induce neuroprotection and functional improvement after ischemic stroke by modulating microglial polarization. Biomaterials science 7(5):2037-2049. doi:10.1039/c8bm01449c

53. Li S, Luo L, He Y, Li R, Xiang Y, Xing Z, Li Y, Albashari AA, Liao X, Zhang K, Gao L, Ye Q (2021) Dental pulp stem cell-derived exosomes alleviate cerebral ischaemia-reperfusion injury through suppressing inflammatory response. Cell proliferation 54(8):e13093. doi:10.1111/cpr.13093

54. Xin H, Li Y, Buller B, Katakowski M, Zhang Y, Wang X, Shang X, Zhang ZG, Chopp M (2012) Exosomemediated transfer of miR-133b from multipotent mesenchymal stromal cells to neural cells contributes to neurite outgrowth. Stem cells 30(7):1556-1564. doi:10.1002/stem.1129

55. Fu C, Li Z, Mao Z (2018) Association between Social Activities and Cognitive Function among the Elderly in China: A Cross-Sectional Study. Int J Environ Res Public Health 15 (2). doi:10.3390/ijerph15020231

56. Kennedy DO (2019) Phytochemicals for Improving Aspects of Cognitive Function and Psychological State Potentially Relevant to Sports Performance. Sports medicine 49(Suppl 1):39-58. doi:10.1007/s40279-018-1007-0

57. Valentine G, Sofuoglu M (2018) Cognitive Effects of Nicotine: Recent Progress. Current neuropharmacology 16(4):403-414. doi:10.2174/1570159X15666171103152136 
58. Birle C, Slavoaca D, Balea M, Livint Popa L, Muresanu I, Stefanescu E, Vacaras V, Dina C, Strilciuc S, Popescu BO, Muresanu DF (2021) Cognitive function: holarchy or holacracy? Neurological sciences: official journal of the Italian Neurological Society and of the Italian Society of Clinical Neurophysiology 42 (1):89-99. doi:10.1007/s10072-020-04737-3

59. Zhang Y, Chopp M, Meng Y, Katakowski M, Xin H, Mahmood A, Xiong Y (2015) Effect of exosomes derived from multipluripotent mesenchymal stromal cells on functional recovery and neurovascular plasticity in rats after traumatic brain injury. Journal of neurosurgery 122(4):856-867. doi:10.3171/2014.11.JNS14770

60. Venkat P, Cui C, Chopp M, Zacharek A, Wang F, Landschoot-Ward J, Shen Y, Chen J (2019) MiR-126 Mediates Brain Endothelial Cell Exosome Treatment-Induced Neurorestorative Effects After Stroke in Type 2 Diabetes Mellitus Mice. Stroke 50(10):2865-2874. doi:10.1161/STROKEAHA.119.025371

61. Niu Y, Wang X, Li M, Niu B (2020) Exosomes from human umbilical cord Mesenchymal stem cells attenuates stress-induced hippocampal dysfunctions. Metabolic brain disease 35(8):1329-1340. doi:10.1007/s11011-019-00514-0

62. Wang Z, Ji C, Wu L, Qiu J, Li Q, Shao Z, Chen G (2014) Tert-butylhydroquinone alleviates early brain injury and cognitive dysfunction after experimental subarachnoid hemorrhage: role of Keap1/Nrf2/ARE pathway. PloS one 9(5):e97685. doi:10.1371/journal.pone.0097685

63. Shang P, Zhang Y, Ma D, Hao Y, Wang X, Xin M, Zhang Y, Zhu M, Feng J (2019) Inflammation resolution and specialized pro-resolving lipid mediators in CNS diseases. Expert opinion on therapeutic targets 23 (11):967-986. doi:10.1080/14728222.2019.1691525

64. Yang QQ, Zhou JW (2019) Neuroinflammation in the central nervous system: Symphony of glial cells. Glia 67(6):1017-1035. doi:10.1002/glia.23571

65. Matsuda M, Huh Y, Ji RR (2019) Roles of inflammation, neurogenic inflammation, and neuroinflammation in pain. Journal of anesthesia 33(1):131-139. doi:10.1007/s00540-018-2579-4

66. Yuan J, Amin P, Ofengeim D (2019) Necroptosis and RIPK1-mediated neuroinflammation in CNS diseases. Nature reviews Neuroscience 20(1):19-33. doi:10.1038/s41583-018-0093-1

67. Linnerbauer M, Wheeler MA, Quintana FJ (2020) Astrocyte Crosstalk in CNS Inflammation. Neuron 108(4):608-622. doi:10.1016/j.neuron.2020.08.012

68. Lai N, Wu D, Liang T, Pan P, Yuan G, Li X, Li H, Shen H, Wang Z, Chen G (2020) Systemic exosomal miR-193b-3p delivery attenuates neuroinflammation in early brain injury after subarachnoid hemorrhage in mice. J Neuroinflamm 17(1):74. doi:10.1186/s12974-020-01745-0

69. Wang K, Ru J, Zhang H, Chen J, Lin X, Lin Z, Wen M, Huang L, Ni H, Zhuge Q, Yang S (2020) Melatonin Enhances the Therapeutic Effect of Plasma Exosomes Against Cerebral Ischemia-Induced Pyroptosis Through the TLR4/NF-kappaB Pathway. Front NeuroSci 14:848. doi:10.3389/fnins.2020.00848

70. Vannella KM, Wynn TA (2017) Mechanisms of Organ Injury and Repair by Macrophages. Annu Rev Physiol 79:593-617. doi:10.1146/annurev-physiol-022516-034356 
71. Dinet V, Petry KG, Badaut J (2019) Brain-Immune Interactions and Neuroinflammation After Traumatic Brain Injury. Front NeuroSci 13:1178. doi:10.3389/fnins.2019.01178

72. Vallon M, Chang J, Zhang H, Kuo CJ (2014) Developmental and pathological angiogenesis in the central nervous system. Cell Mol Life Sci 71(18):3489-3506. doi:10.1007/s00018-014-1625-0

73. Ma S, Huang Z (2015) Neural Regulation of CNS Angiogenesis During Development. Frontiers in biology 10(1):61-73. doi:10.1007/s11515-014-1331-y

74. Melincovici CS, Bosca AB, Susman S, Marginean M, Mihu C, Istrate M, Moldovan IM, Roman AL, Mihu CM (2018) Vascular endothelial growth factor (VEGF) - key factor in normal and pathological angiogenesis. Romanian journal of morphology embryology = Revue roumaine de morphologie et embryologie 59(2):455-467

75. Apte RS, Chen DS, Ferrara N (2019) VEGF in Signaling and Disease: Beyond Discovery and Development. Cell 176(6):1248-1264. doi:10.1016/j.cell.2019.01.021

76. Hou K, Li G, Zhao J, Xu B, Zhang Y, Yu J, Xu K (2020) Bone mesenchymal stem cell-derived exosomal microRNA-29b-3p prevents hypoxic-ischemic injury in rat brain by activating the PTEN-mediated Akt signaling pathway. J Neuroinflamm 17(1):46. doi:10.1186/s12974-020-1725-8

77. Zhang Y, Zhang Y, Chopp M, Pang H, Zhang ZG, Mahmood A, Xiong Y (2021) MiR-17-92 ClusterEnriched Exosomes Derived from Human Bone Marrow Mesenchymal Stromal Cells Improve Tissue and Functional Recovery in Rats after Traumatic Brain Injury. J Neurotrauma 38(11):1535-1550. doi:10.1089/neu.2020.7575

78. Quillinan N, Herson PS, Traystman RJ (2016) Neuropathophysiology of Brain Injury. Anesthesiol Clin 34(3):453-464. doi:10.1016/j.anclin.2016.04.011

79. Rodriguez J, Li T, Xu Y, Sun Y, Zhu C (2021) Role of apoptosis-inducing factor in perinatal hypoxicischemic brain injury. Neural regeneration research 16(2):205-213. doi:10.4103/1673-5374.290875

80. Wang XX, Zhang B, Xia R, Jia QY (2020) Inflammation, apoptosis and autophagy as critical players in vascular dementia. Eur Rev Med Pharmacol Sci 24(18):9601-9614.

doi:10.26355/eurrev_202009_23048

81. Radak D, Katsiki N, Resanovic I, Jovanovic A, Sudar-Milovanovic E, Zafirovic S, Mousad SA, Isenovic ER (2017) Apoptosis and Acute Brain Ischemia in Ischemic Stroke. Curr Vasc Pharmacol 15(2):115122. doi:10.2174/1570161115666161104095522

82. Bruggeman GF, Haitsma IK, Dirven CMF, Volovici V (2021) Traumatic axonal injury (TAI): definitions, pathophysiology and imaging-a narrative review. Acta Neurochir 163(1):31-44. doi:10.1007/s00701020-04594-1

83. Song Y, Li Z, He T, Qu M, Jiang L, Li W, Shi X, Pan J, Zhang L, Wang Y, Zhang Z, Tang Y, Yang GY (2019) M2 microglia-derived exosomes protect the mouse brain from ischemia-reperfusion injury via exosomal miR-124. Theranostics 9(10):2910-2923. doi:10.7150/thno.30879

84. Ni H, Yang S, Siaw-Debrah F, Hu J, Wu K, He Z, Yang J, Pan S, Lin X, Ye H, Xu Z, Wang F, Jin K, Zhuge Q, Huang L (2019) Exosomes Derived From Bone Mesenchymal Stem Cells Ameliorate Early 
Inflammatory Responses Following Traumatic Brain Injury. Front NeuroSci 13:14. doi:10.3389/fnins.2019.00014

85. Xu X, Lai Y, Hua ZC (2019) Apoptosis and apoptotic body: disease message and therapeutic target potentials. Bioscience reports 39 (1). doi:10.1042/BSR20180992

86. Obeng E (2021) Apoptosis (programmed cell death) and its signals - A review. Brazilian journal of biology = Revista brasleira de biologia 81(4):1133-1143. doi:10.1590/1519-6984.228437

87. Levine B, Kroemer G (2019) Biological Functions of Autophagy Genes: A Disease Perspective. Cell 176(1-2):11-42. doi:10.1016/j.cell.2018.09.048

88. Parzych KR, Klionsky DJ (2014) An overview of autophagy: morphology, mechanism, and regulation. Antioxid Redox Signal 20(3):460-473. doi:10.1089/ars.2013.5371

89. Saha S, Panigrahi DP, Patil S, Bhutia SK (2018) Autophagy in health and disease: A comprehensive review. Biomedicine pharmacotherapy $=$ Biomedecine pharmacotherapie 104:485-495. doi:10.1016/j.biopha.2018.05.007

90. Zeng Z, Zhang Y, Jiang W, He L, Qu H (2020) Modulation of autophagy in traumatic brain injury. Journal of cellular physiology 235(3):1973-1985. doi:10.1002/jcp.29173

91. Shi CX, Jin J, Wang XQ, Song T, Li GH, Li KZ, Ma JH (2020) Sevoflurane attenuates brain damage through inhibiting autophagy and apoptosis in cerebral ischemiareperfusion rats. Mol Med Rep 21(1):123-130. doi:10.3892/mmr.2019.10832

92. Ahsan A, Zheng YR, Wu XL, Tang WD, Liu MR, Ma SJ, Jiang L, Hu WW, Zhang XN, Chen Z (2019) Urolithin A-activated autophagy but not mitophagy protects against ischemic neuronal injury by inhibiting ER stress in vitro and in vivo. CNS Neurosci Ther 25(9):976-986. doi:10.1111/cns.13136

93. Li D, Huang S, Zhu J, Hu T, Han Z, Zhang S, Zhao J, Chen F, Lei P (2019) Exosomes from MiR-21-5pIncreased Neurons Play a Role in Neuroprotection by Suppressing Rab11a-Mediated Neuronal Autophagy In Vitro After Traumatic Brain Injury. Medical science monitor: international medical journal of experimental clinical research 25:1871-1885. doi:10.12659/MSM.915727

94. Yuan FY, Zhang MX, Shi YH, Li MH, Ou JY, Bai WF, Zhang MS (2020) Bone marrow stromal cellsderived exosomes target DAB2IP to induce microglial cell autophagy, a new strategy for neural stem cell transplantation in brain injury. Experimental therapeutic medicine 20(3):2752-2764. doi:10.3892/etm.2020.9008

95. Sweeney MD, Zhao Z, Montagne A, Nelson AR, Zlokovic BV (2019) Blood-Brain Barrier: From Physiology to Disease and Back. Physiological reviews 99(1):21-78. doi:10.1152/physrev.00050.2017

96. Jiang X, Andjelkovic AV, Zhu L, Yang T, Bennett MVL, Chen J, Keep RF, Shi Y (2018) Blood-brain barrier dysfunction and recovery after ischemic stroke. Progress in neurobiology 163-164:144-171. doi:10.1016/j.pneurobio.2017.10.001

97. Langen UH, Ayloo S, Gu C (2019) Development and Cell Biology of the Blood-Brain Barrier. Annu Rev Cell Dev Biol 35:591-613. doi:10.1146/annurev-cellbio-100617-062608 
98. Pan Q, Kuang X, Cai S, Wang X, Du D, Wang J, Wang Y, Chen Y, Bihl J, Chen Y, Zhao B, Ma X (2020) miR-132-3p priming enhances the effects of mesenchymal stromal cell-derived exosomes on ameliorating brain ischemic injury. Stem Cell Res Ther 11(1):260. doi:10.1186/s13287-020-01761-0

99. Zhang L, Liu H, Jia L, Lyu J, Sun Y, Yu H, Li H, Liu W, Weng Y, Yu W (2019) Exosomes Mediate Hippocampal and Cortical Neuronal Injury Induced by Hepatic Ischemia-Reperfusion Injury through Activating Pyroptosis in Rats. Oxidative medicine and cellular longevity 2019:3753485. doi:10.1155/2019/3753485

100. Tafrihi M, Hasheminasab E (2019) MiRNAs: Biology, Biogenesis, their Web-based Tools, and Databases. MicroRNA 8(1):4-27. doi:10.2174/2211536607666180827111633

101. Gjorgjieva M, Sobolewski C, Dolicka D, Correia de Sousa M, Foti M (2019) miRNAs and NAFLD: from pathophysiology to therapy. Gut 68(11):2065-2079. doi:10.1136/gutjnl-2018-318146

102. Saliminejad K, Khorram Khorshid HR, Soleymani Fard S, Ghaffari SH (2019) An overview of microRNAs: Biology, functions, therapeutics, and analysis methods. Journal of cellular physiology 234(5):5451-5465. doi:10.1002/jcp.27486

103. Mori MA, Ludwig RG, Garcia-Martin R, Brandao BB, Kahn CR (2019) Extracellular miRNAs: From Biomarkers to Mediators of Physiology and Disease. Cell Metabol 30(4):656-673. doi:10.1016/j.cmet.2019.07.011

104. Lu TX, Rothenberg ME (2018) MicroRNA. J Allergy Clin Immunol 141(4):1202-1207. doi:10.1016/j.jaci.2017.08.034

105. Yue B, Yang H, Wang J, Ru W, Wu J, Huang Y, Lan X, Lei C, Chen H (2020) Exosome biogenesis, secretion and function of exosomal miRNAs in skeletal muscle myogenesis. Cell proliferation 53(7):e12857. doi:10.1111/cpr.12857

106. Sun Z, Shi K, Yang S, Liu J, Zhou Q, Wang G, Song J, Li Z, Zhang Z, Yuan W (2018) Effect of exosomal miRNA on cancer biology and clinical applications. Mol Cancer 17(1):147. doi:10.1186/s12943-018-0897-7

107. Williams LM, Gilmore TD (2020) Looking Down on NF-kappaB. Molecular cellular biology 40 (15). doi:10.1128/MCB.00104-20

108. Choi MC, Jo J, Park J, Kang HK, Park Y (2019) NF-kappaB Signaling Pathways in Osteoarthritic Cartilage Destruction. Cells 8 (7). doi:10.3390/cells8070734

109. Jimi E, Fei H, Nakatomi C (2019) NF-kappaB Signaling Regulates Physiological and Pathological Chondrogenesis. Int J Mol Sci 20 (24). doi:10.3390/ijms20246275

110. Taniguchi K, Karin M (2018) NF-kappaB, inflammation, immunity and cancer: coming of age. Nature reviews Immunology 18(5):309-324. doi:10.1038/nri.2017.142

111. Lu Y, Zhou Y, Zhang R, Wen L, Wu K, Li Y, Yao Y, Duan R, Jia Y (2019) Bone Mesenchymal Stem CellDerived Extracellular Vesicles Promote Recovery Following Spinal Cord Injury via Improvement of the Integrity of the Blood-Spinal Cord Barrier. Front NeuroSci 13:209. doi:10.3389/fnins.2019.00209

112. Zhu B, Zhang L, Liang C, Liu B, Pan X, Wang Y, Zhang Y, Zhang Y, Xie W, Yan B, Liu F, Yip HK, Yu XY, Li Y (2019) Stem Cell-Derived Exosomes Prevent Aging-Induced Cardiac Dysfunction through a Novel 
Exosome/IncRNA MALAT1/NF-kappaB/TNF-alpha Signaling Pathway. Oxidative medicine and cellular longevity 2019:9739258. doi:10.1155/2019/9739258

113. Liu B, Sun L, Liu Q, Gong C, Yao Y, Lv X, Lin L, Yao H, Su F, Li D, Zeng M, Song E (2015) A cytoplasmic NF-kappaB interacting long noncoding RNA blocks IkappaB phosphorylation and suppresses breast cancer metastasis. Cancer cell 27(3):370-381. doi:10.1016/j.ccell.2015.02.004

114. Cao Y, Tang S, Nie X, Zhou Z, Ruan G, Han W, Zhu Z, Ding C (2021) Decreased miR-214-3p activates NF-kappaB pathway and aggravates osteoarthritis progression. EBioMedicine 65:103283. doi:10.1016/j.ebiom.2021.103283

115. Xie Y, Shi X, Sheng K, Han G, Li W, Zhao Q, Jiang B, Feng J, Li J, Gu Y (2019) PI3K/Akt signaling transduction pathway, erythropoiesis and glycolysis in hypoxia (Review). Mol Med Rep 19(2):783791. doi:10.3892/mmr.2018.9713

116. Xu F, Na L, Li Y, Chen L (2020) Roles of the PI3K/AKT/mTOR signalling pathways in neurodegenerative diseases and tumours. Cell bioscience 10:54. doi:10.1186/s13578-020-00416-0

117. Tsai CY, Dai KY, Fang C, Wu JCC, Chan SHH (2018) PTEN/FLJ10540/PI3K/Akt cascade in experimental brain stem death: A newfound role for a classical tumorigenic signaling pathway. Biochem Pharmacol 155:207-212. doi:10.1016/j.bcp.2018.07.002

118. Hu M, Zhu S, Xiong S, Xue X, Zhou X (2019) MicroRNAs and the PTEN/PI3K/Akt pathway in gastric cancer (Review). Oncol Rep 41(3):1439-1454. doi:10.3892/or.2019.6962

119. Chen H, Zhou L, Wu X, Li R, Wen J, Sha J, Wen X (2016) The PI3K/AKT pathway in the pathogenesis of prostate cancer. Frontiers in bioscience 21:1084-1091. doi:10.2741/4443

120. Papa A, Pandolfi PP (2019) The PTEN(-)PI3K Axis in Cancer. Biomolecules 9 (4). doi:10.3390/biom9040153

121. Zhao S, Mi Y, Guan B, Zheng B, Wei P, Gu Y, Zhang Z, Cai S, Xu Y, Li X, He X, Zhong X, Li G, Chen Z, Li D (2020) Tumor-derived exosomal miR-934 induces macrophage M2 polarization to promote liver metastasis of colorectal cancer. J Hematol Oncol 13(1):156. doi:10.1186/s13045-020-00991-2

122. Fu X, Liu M, Qu S, Ma J, Zhang Y, Shi T, Wen H, Yang Y, Wang S, Wang J, Nan K, Yao Y, Tian T (2018) Exosomal microRNA-32-5p induces multidrug resistance in hepatocellular carcinoma via the PI3K/Akt pathway. Journal of experimental clinical cancer research: CR 37(1):52. doi:10.1186/s13046-018-0677-7

123. Michelini S, Ricci M, Serrani R, Barati S, Kenanoglu S, Veselenyiova D, Kurti D, Baglivo M, Basha SH, Priya S, Dautaj A, Dundar M, Krajcovic J, Bertelli M (2021) NOTCH1: Review of its role in lymphatic development and study of seven families with rare pathogenic variants. Mol Genet Genom Med 9(1):e1529. doi:10.1002/mgg3.1529

124. Gharaibeh L, Elmadany N, Alwosaibai K, Alshaer W (2020) Notch1 in Cancer Therapy: Possible Clinical Implications and Challenges. Mol Pharmacol 98(5):559-576. doi:10.1124/molpharm.120.000006

125. Arumugam TV, Baik SH, Balaganapathy P, Sobey CG, Mattson MP, Jo DG (2018) Notch signaling and neuronal death in stroke. Progress in neurobiology 165-167:103-116. 
doi:10.1016/j.pneurobio.2018.03.002

126. Liang W, Lin C, Yuan L, Chen L, Guo P, Li P, Wang W, Zhang X (2019) Preactivation of Notch1 in remote ischemic preconditioning reduces cerebral ischemia-reperfusion injury through crosstalk with the NF-kappaB pathway. J Neuroinflamm 16(1):181. doi:10.1186/s12974-019-1570-9

127. Zhang D, Cai G, Liu K, Zhuang Z, Jia K, Pei S, Wang X, Wang H, Xu S, Cui C, Sun M, Guo S, Song W, Cai G (2021) Microglia exosomal miRNA-137 attenuates ischemic brain injury through targeting Notch1. Aging 13(3):4079-4095. doi:10.18632/aging.202373

128. Liu S, Liu D, Chen C, Hamamura K, Moshaverinia A, Yang R, Liu Y, Jin Y, Shi S (2015) MSC Transplantation Improves Osteopenia via Epigenetic Regulation of Notch Signaling in Lupus. Cell Metabol 22(4):606-618. doi:10.1016/j.cmet.2015.08.018

129. Engler A, Zhang R, Taylor V (2018) Notch and Neurogenesis. Adv Exp Med Biol 1066:223-234. doi:10.1007/978-3-319-89512-3_11

130. Zanotti S, Canalis E (2016) Notch Signaling and the Skeleton. Endocr Rev 37(3):223-253. doi:10.1210/er.2016-1002

131. Wang JQ, Mao L (2019) The ERK Pathway: Molecular Mechanisms and Treatment of Depression. Mol Neurobiol 56(9):6197-6205. doi:10.1007/s12035-019-1524-3

132. Rai SN, Dilnashin H, Birla H, Singh SS, Zahra W, Rathore AS, Singh BK, Singh SP (2019) The Role of PI3K/Akt and ERK in Neurodegenerative Disorders. Neurotox Res 35(3):775-795. doi:10.1007/s12640-019-0003-y

133. Vithayathil J, Pucilowska J, Landreth GE (2018) ERK/MAPK signaling and autism spectrum disorders. Prog Brain Res 241:63-112. doi:10.1016/bs.pbr.2018.09.008

134. Long X, Yao X, Jiang Q, Yang Y, He X, Tian W, Zhao K, Zhang H (2020) Astrocyte-derived exosomes enriched with miR-873a-5p inhibit neuroinflammation via microglia phenotype modulation after traumatic brain injury. J Neuroinflamm 17(1):89. doi:10.1186/s12974-020-01761-0

135. Nakamura Y, Park JH, Hayakawa K (2020) Therapeutic use of extracellular mitochondria in CNS injury and disease. Exp Neurol 324:113114. doi:10.1016/j.expneurol.2019.113114

136. Ladak AA, Enam SA, Ibrahim MT (2019) A Review of the Molecular Mechanisms of Traumatic Brain Injury. World neurosurgery 131:126-132. doi:10.1016/j.wneu.2019.07.039

137. Binvignat O, Olloquequi J (2020) Excitotoxicity as a Target Against Neurodegenerative Processes. Curr Pharm Design 26(12):1251-1262. doi:10.2174/1381612826666200113162641

138. Iovino L, Tremblay ME, Civiero L (2020) Glutamate-induced excitotoxicity in Parkinson's disease: The role of glial cells. J Pharmacol Sci 144(3):151-164. doi:10.1016/j.jphs.2020.07.011

139. Yakovlev AA, Lyzhin AA, Aleksandrova OP, Khaspekov LG, Gulyaeva NV (2019) [Exosomes secretion and autophagy in long-term protection of neurons from excitotoxic damage]. Biomeditsinskaia khimiia 65(5):361-365. doi:10.18097/PBMC20196505361

140. Shakespear N, Ogura M, Yamaki J, Homma Y (2020) Astrocyte-Derived Exosomal microRNA miR200a-3p Prevents MPP(+)-Induced Apoptotic Cell Death Through Down-Regulation of MKK4. 
Neurochem Res 45(5):1020-1033. doi:10.1007/s11064-020-02977-5

141. Eitan E, Hutchison ER, Marosi K, Comotto J, Mustapic M, Nigam SM, Suire C, Maharana C, Jicha GA, Liu D, Machairaki V, Witwer KW, Kapogiannis D, Mattson MP (2016) Extracellular Vesicle-Associated Abeta Mediates Trans-Neuronal Bioenergetic and $\mathrm{Ca}(2+)$-Handling Deficits in Alzheimer's Disease Models. NPJ aging and mechanisms of disease 2. doi:10.1038/npjamd.2016.19

142. Zhang L, Wang H (2018) Targeting the NF-E2-Related Factor 2 Pathway: a Novel Strategy for Traumatic Brain Injury. Mol Neurobiol 55(2):1773-1785. doi:10.1007/s12035-017-0456-z

143. Khatri N, Thakur M, Pareek V, Kumar S, Sharma S, Datusalia AK (2018) Oxidative Stress: Major Threat in Traumatic Brain Injury. CNS Neurol Disord Drug Target 17(9):689-695. doi:10.2174/1871527317666180627120501

144. Li P, Stetler RA, Leak RK, Shi Y, Li Y, Yu W, Bennett MVL, Chen J (2018) Oxidative stress and DNA damage after cerebral ischemia: Potential therapeutic targets to repair the genome and improve stroke recovery. Neuropharmacology 134 (Pt B):208-217. doi:10.1016/j.neuropharm.2017.11.011

145. Rodriguez-Rodriguez A, Egea-Guerrero JJ, Murillo-Cabezas F, Carrillo-Vico A (2014) Oxidative stress in traumatic brain injury. Curr Med Chem 21(10):1201-1211

146. Wang T, Jian Z, Baskys A, Yang J, Li J, Guo H, Hei Y, Xian P, He Z, Li Z, Li N, Long Q (2020) MSCderived exosomes protect against oxidative stress-induced skin injury via adaptive regulation of the NRF2 defense system. Biomaterials 257:120264. doi:10.1016/j.biomaterials.2020.120264

147. Arslan F, Lai RC, Smeets MB, Akeroyd L, Choo A, Aguor EN, Timmers L, van Rijen HV, Doevendans PA, Pasterkamp G, Lim SK, de Kleijn DP (2013) Mesenchymal stem cell-derived exosomes increase ATP levels, decrease oxidative stress and activate PI3K/Akt pathway to enhance myocardial viability and prevent adverse remodeling after myocardial ischemia/reperfusion injury. Stem cell research 10(3):301-312. doi:10.1016/j.scr.2013.01.002

148. Perut F, Roncuzzi L, Avnet S, Massa A, Zini N, Sabbadini S, Giampieri F, Mezzetti B, Baldini N (2021) Strawberry-Derived Exosome-Like Nanoparticles Prevent Oxidative Stress in Human Mesenchymal Stromal Cells. Biomolecules 11 (1). doi:10.3390/biom11010087

\section{Figures}




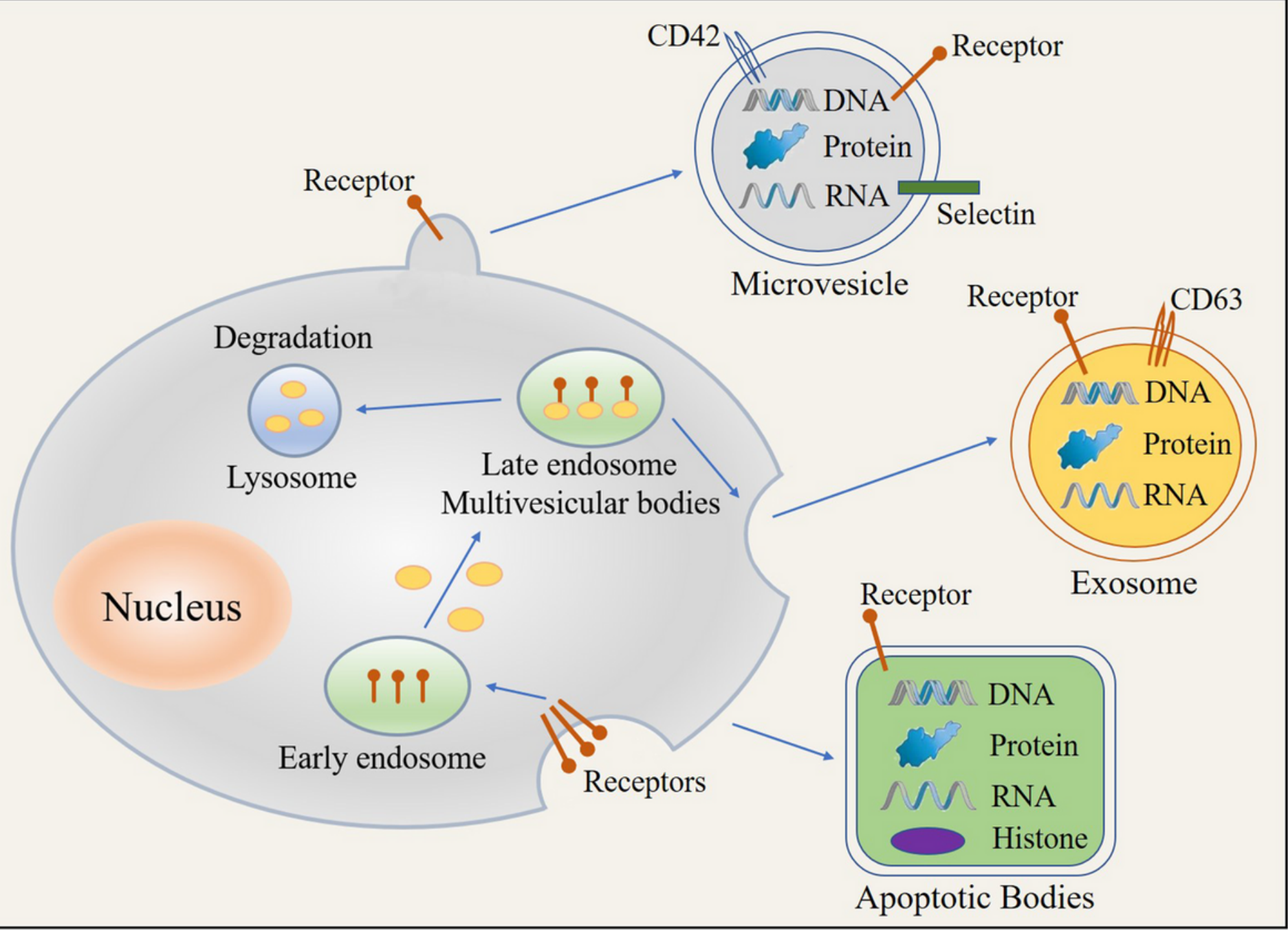

Figure 1

The biogenesis of extracellular vesicles. Exosomes containing DNAs, RNAs and proteins firstly fuse into early endosomes to form late endosomes (multivesicular bodies, MVBs). Late MVBs then fuse with lysosomes for degradation or fuse with plasma membrane to release exosomes. Microvesicles are secreted by outward budding and splitting of plasma membrane. Apoptotic bodies are produced by blebbing of apoptotic cells. They contain DNAs, RNAs, proteins and histone. 


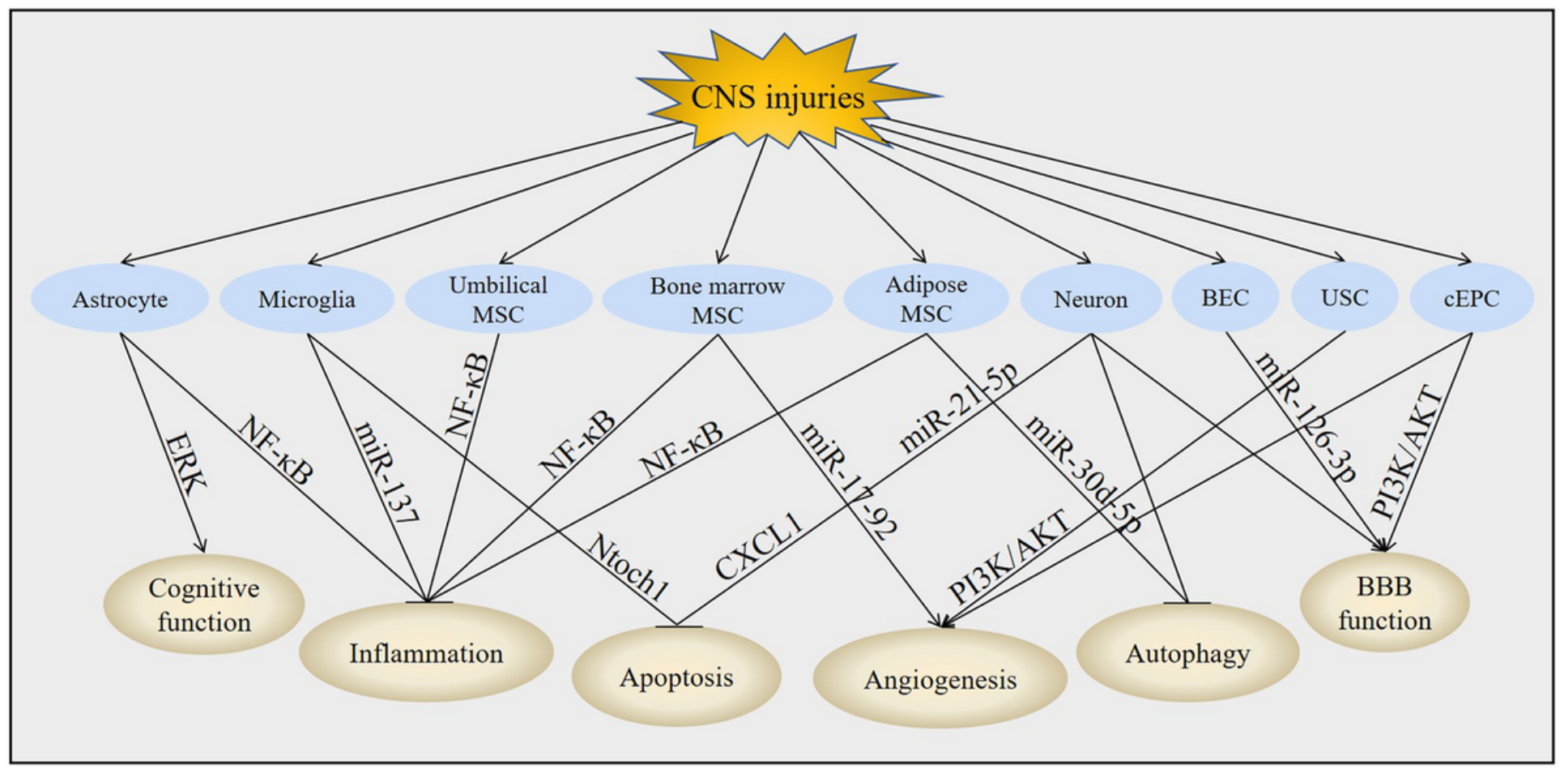

Figure 2

Downstream molecules of exosomes in CNS injuries. In CNS injuries, astrocyte, microglia, umbilical MSC, bone marrow MSC, adipose MSC, neuron, BEC, USC and CEPC-derived exosomes lead to the modulation of miRNAs, activation of PI3K/AKT and inhibition of ERK, NF-KB, Notch1, CXCL1. These downstream molecules subsequently improved cognitive function, promoted angiogenesis, regulated autophagy, protected BBB function, and suppressed apoptosis and inflammation post-CNS injuries. 